Available online at http://bjas.bajas.edu.iq

https://doi.org/10.37077/25200860.2020.32.1.04

College of Agriculture, University of Basrah

Basrah J. Agric. Sci., 33(1): 39-49, 2020

\section{Basrah Journal of Agricultural Sciences}

E-ISSN: 2520-0860

ISSN $1814-5868$

\title{
A Novel Report on Killer Yeast Strains Identification Methods
}

\author{
Najwa M.J.A. Abu-Mejdad 1*, Adnan I. Al-Badran², Abdullah H. Al-Saadoon ${ }^{3}$ \\ ${ }^{1,2}$ Department of Biology, College of Science, University of Basrah, Basrah, Iraq. \\ ${ }^{3}$ Department of Pathological Analyses, College of Science, University of Basrah, Iraq \\ *Corresponding author e-mail: najwa_22_4_1978@yahoo.com \\ Received 19 September 2019; Accepted 31 December 2019; Available online 24 June 2019
}

\begin{abstract}
This study was conducted to isolate and identify killer yeasts from soil samples that collected from different locations in Basrah and Dhi-Qar provinces. Seventyfive soil samples were collected from different areas, including sandy, arable, surface sediment and uncultivated soil, using dilution methods to cultivate a serial dilution of each soil sample. The results showed that a 112 isolates were identified biochemically using VITEK system and molecularly using internal transcribed spacer (ITS1- 5.8S-ITS2) marker. The molecular identification provided fast and precise identification results for the 112 isolates, whereas the VITEK test resulted low identification efficiency ( $8.2 \%$ were accurate and $91.8 \%$ were not). The Diazonium blue B salts produced a good colour reaction in distinguishing between ascomycetes and basidiomycetes. The PCR was more accurate in identification of killer yeasts compared to the VITEK system.
\end{abstract}

Key words: Diazonium blue B, VITEK, PCR, Killer yeasts, Diagnosis.

\section{Introduction}

The morphological, biochemical and molecular identification of killer yeasts have been carried out in comprehensive studies and various identifications. The killer yeasts can be grouped into three categories based on genetic determinants that encoding the killer activities that are either cytoplasmically inherited encapsulated dsRNA viruses, linear dsDNA plasmids or nuclear genes (Schmitt \& Breinig, 2002). According to the molecular identification, each killer toxin has been found to possess its own novel system for toxicity .(Magliani et al., 1997).
However, the morphological identification methods are not enough to be considered as a single yeast identification tool. This means that these methods require high experience due to unstable or variable characteristics. For example, the Diazonium blue B salt as a morphological test is considered good in discriminating between basidiomycetes species that showing red colour colony when reacting against this salt, and the ascomycetes yeast presented a brown colour colony (negative reaction). This salt therefore can be 
considered as a good test to differentiate between the groups of fungi depending on the different colour consequences. This difference of colony colours can be attributed to different components in cell wall of ascomycetes and basidiomycetes, and also depending on experience of lab technicians (Van der Walt, 1976. , Rodrigues, 2018) Thus, the mycologists use biochemical identification to achieve accurate identification of yeast, such as commercial miniaturized systems, VITEK, API 32C and API 20C AUX from BioMerieux, which are not in common but also fast, effective and commercially available biochemical systems. When combining biochemical profiles that can be obtained by using systems such as the VITEK yeast biochemical card (YBC) (Latouche et al., 1997) and the various API kits (i.e., 20C and ID 32C) to computerized databases, their use in rapid yeast identification has become more interesting tests that require less time and technical expertise compared to the morphological criteria. However, biochemical profiles can be varied due to their minor changes in test conditions. Accordingly, several reports have indicated needs of confirming identification by combining the biochemical profiles and morphological observations (Török \& King, 1991). Even though, most diagnostic laboratories still rely on the conventional or traditional methods in identification of yeast species as the mainstay of species identification, but these methods are difficult, time-consuming and can lead to wrong identification. On the other hand, progress in molecular biology has provided a large number of DNA based techniques for identifying and characterizing yeasts (Hierro et al., 2004). Moreover, molecular techniques have become increasingly used as tools for yeast identification, especially when dealing with species that their morphological features are difficult to distinguish. The applications of these techniques have generated a greater number of studies on the classification, identification and ecology of yeast species (Tekpinar \& Kalmer, 2019). This study aimed to identify yeasts using morphological, biochemical and molecular methods.

\section{Materials \& Methods}

\section{Sample collection}

During this study, 75 soil samples were collected from sandy, arable, surface sediment and uncultivated soil, and the dilution method was used for fungal isolation.

\section{Diazonium blue B stain (DBB stain)}

The DBB stain was prepared from dissolving $0.1 \mathrm{gm}$ of $\mathrm{DBB}$ salt in $100 \mathrm{ml}$ of Tris- $\mathrm{HCl}$ buffer $(0.1 \mathrm{M})$.

\subsection{Tris HCl (1 X)}

The buffer was prepared by dissolving 1.211 gm of Tris HCL in $80 \mathrm{ml}$ of D.W. then the volume was completed to $100 \mathrm{ml}$ and the $\mathrm{pH}$ value was adjusted to 7.0 (Dufour et al., 2003).

\section{Diazonium blue B test on solid media}

The yeast isolates were cultured on SDA $0.5 \%$ yeast extract, incubated for 7 days at $25{ }^{\circ} \mathrm{C}$, then 1-2 drops of stain was added to the culture after 2-3 days of incubation. The pink or violet colour was considered as a positive, result while the brown colour was considered as a negative (Hagler \& Ahearn, 1981).

\section{Isolation of yeasts}

One gram of soil from each sample was added to a sterile $15 \mathrm{ml}$ test tube containing $9 \mathrm{ml}$ of different solvents (peptone water 0.1 $\%$, distilled water, phosphate buffered saline, normal saline) then mixing by vortex for 15 minutes to give $1 / 10$ dilution 1 (D1). Serial dilution of $10^{-1}-10^{-3}$ was performed, and one $\mathrm{ml}$ of each dilution was culture on 
solid media then incubated at $25^{\circ} \mathrm{C}$ for 72 hours.

\section{Biochemical identification}

The biochemical identification of yeast colonies was performed by VITEK 2 system (Biomerieux, USA) using yeast biochemical card (YBC) and according to the manufacturer instructions.

Molecular study

DNA extraction
The DNA was extracted from yeast isolate according to the procedure of Presto ${ }^{\mathrm{TM}}$ Mini gDNA Yeast Kit (Geneaid, Taiwan).

\section{Polymerase chain reaction $(\mathrm{PCR})$}

The PCR technique was used to amplify the internal transcribed spacer (ITS1- 5.8S-ITS2) region for 134 isolates using two universal primers (ITS1 and ITS4) for amplification (Bellemain et al., 2010)

\begin{tabular}{|l|l|l|}
\hline Primer & \multicolumn{1}{|c|}{ Primer sequences (5'- 3') } & Length \\
\hline ITS1 & F-5-TCC GTA GGT GAA CCT GCG G-3 & 19 base \\
\hline ITS4 & R-5-TCC TCC GCT TAT TGA TAT GC-3 & 20 base \\
\hline
\end{tabular}

Sequencing of ITS1- 5.8S - ITS2 rDNA Gene

\section{Sample preparation for sequencing}

Twenty $\mu 1$ of ITS1-5.8S-ITS2 rDNA PCR product of each sample was send to Macrogen company, Korea (http://dna.macrogen.com) for sequencing.

\section{Identification of yeasts species}

The sequence results of yeasts species were analyzed using basic local alignment search tool (BLAST) to compare our sequences with deposited copies in National Center for Biotechnology Information (NCBI) (http://www.ncbi.nlm.nih.gov).

\section{Results}

The morphological, biochemical tests and molecular diagnosis results (Tables 1 and 2; fig. 1) revealed that the identified isolates were belonged to 112 type strains.

\section{Diazonium Blue B (DBB) test to detect basidiomycetous yeasts}

The most cells of basidiomycetous yeasts showed pink to red or violet colour reactions against DBB when using many solid growth media. However, there were great differences among isolates in the colour intensity and culture ages, which were necessary for obtaining the positive reaction. Rhodotorula mucilaginosa showed a positive results after twenty four hour at $25^{\circ} \mathrm{C}$, whereas Filobasidium oeirense took three weeks to give a positive results. In contrast, some basidiomycetes got a negative results, such as Symmetrospora folicola, Vishniacozyma carnescens, Cystobasidium benthicum and Cystobasidium minutum. On the other hand, the most ascomycetous yeasts presented negative results, except Candida membranifaciens and Candida glabrata (Fig. 1, Table 1). 
Abu-Mejdad et al. / Basrah J. Agric. Sci., 33(1): 39-49, 2020

Table (1): DBB reaction test to distinguish between basidiomycetous yeasts and ascomycetous yeasts.

\begin{tabular}{|c|c|c|}
\hline No. & Species & DBB reaction \\
\hline 1 & Aureobasidium melanogenum & - \\
\hline 2 & Cadida tropicalis & - \\
\hline 3 & C. membranifaciens & 2 \\
\hline 4 & C. glabrata & 2 \\
\hline 5 & Cutaneotrichosporon dermatis & 2 \\
\hline 6 & Cystobasidium benthicum & - \\
\hline 7 & C. minutum & - \\
\hline 8 & Debaryomyces hansenii & - \\
\hline 9 & Filobasidium oeirense & 3 \\
\hline 10 & Galactomyces pseudocandidum & - \\
\hline 11 & G. reessii & - \\
\hline 12 & Geotrichum candidum & - \\
\hline 13 & Hanseniaspora uvarum & - \\
\hline 14 & Lodderomyces elongisporus & - \\
\hline 15 & Meyerozyma caribbica & - \\
\hline 16 & Naganishia adeliensis & 1 \\
\hline 17 & N. albida & 1 \\
\hline 18 & N. albidosimilis & 2 \\
\hline 19 & N.diffluens & 3 \\
\hline 20 & N. liquefaciens & 3 \\
\hline 21 & N. uzbekistanensis & 3 \\
\hline 22 & N. vishniacil & 2 \\
\hline 23 & Pichia fermentans & - \\
\hline 24 & Rhodotorula diobovata & 2 \\
\hline 25 & R. mucilaginosa & 3 \\
\hline 26 & Symmetrospora folicola & - \\
\hline 27 & Torulaspora delbrueckii & - \\
\hline 28 & Vishniacozyma carnescens & - \\
\hline 29 & Wickerhamomyces anomalus & - \\
\hline 30 & Wickerhamomyces onychis & - \\
\hline 31 & Yarrowia lipolytica & - \\
\hline
\end{tabular}

(Positive results): 1, Pink violet; 2, violet; 3, dark violet to purple (negative results) yellow or brown

Note: Highlighted yellow colour means basidiomycetes that gave negative results in DBB test, while highlighted green colour means ascomycetes that gave positive results in violate colour on 2 solid media as a result of DBB test. 
Abu-Mejdad et al. / Basrah J. Agric. Sci., 33(1): 39-49, 2020
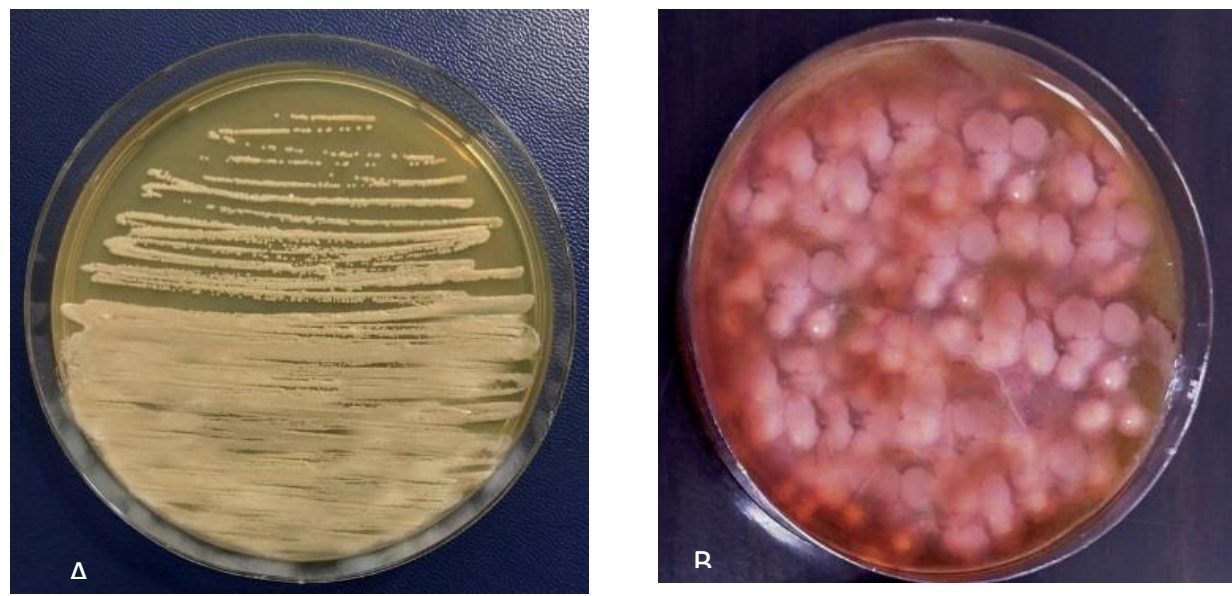

Fig. (1): DBB test (A): Wickerhamomyces anomalus - ve (B): Rhodotorula mucilaginosa +ve (3).

\section{Comparison between yeast identification by} VITEK 2 system and ITS1-ITS2 rDNA gene Sequencing

The comparison between VITEK and molecular identification technique (Table 2) to determine the species level of 112 yeast isolates was not identical. Only $8.2 \%(11$ isolates, No. 11, 62, 72, 75, 160, 164, 203, 215, 234, 246 and 253) were identified as Candida tropicalis by both techniques, while the rest $(91.8 \%)$ were completely mismatched.

Table (2): A comparison between VITEK and ITS1-ITS2 rDNA gene identification.

\begin{tabular}{|c|l|c|l|c|}
\hline Isolates & Species name in gene bank & Homology & VITEK & Identification \\
\hline 102 & Aureobasidium melanogenum & $100 \%$ & Un-identified & \\
\hline 87 & Candida membranifaciens & $100 \%$ & Un-identified & \\
\hline 170 & C. membranifaciens & $100 \%$ & Un-identified & \\
\hline 236 & C. membranifaciens & $100 \%$ & Un-identified & \\
\hline 11 & C. tropicalis & $100 \%$ & Candida tropicalis & $96 \%$ \\
\hline 62 & C. tropicalis & $100 \%$ & C. tropicalis & $96 \%$ \\
\hline 72 & C. tropicalis & $100 \%$ & C. tropicalis & $96 \%$ \\
\hline 75 & C. tropicalis & $100 \%$ & C. tropicalis & $96 \%$ \\
\hline 158 & C. tropicalis & $100 \%$ & C. tropicalis & $96 \%$ \\
\hline 164 & C. tropicalis & $100 \%$ & C. tropicalis & $96 \%$ \\
\hline 203 & C. tropicalis & $100 \%$ & C. tropicalis & $96 \%$ \\
\hline 215 & C. tropicalis & $100 \%$ & C. tropicalis & $96 \%$ \\
\hline 234 & C. tropicalis & $100 \%$ & C. tropicalis & $96 \%$ \\
\hline 246 & C. tropicalis & $100 \%$ & C. tropicalis & $96 \%$ \\
\hline 253 & C. tropicalis & $100 \%$ & C. tropicalis & $96 \%$ \\
\hline 160 & C. glabrata & $100 \%$ & C. tropicalis & $94 \%$ \\
\hline 49 & C. membranifaciens & $100 \%$ & Un-identified & \\
\hline 173 & C. membranifaciens & $100 \%$ & Un-identified & \\
\hline 188 & Cryptococcus albidisimilis & $99 \%$ & Cryptococcus & $97 \%$ \\
\hline 134 & C. diffluens & $100 \%$ & Cryptococcus & $93 \%$ \\
\hline 218 & C. uzbekistanensis & $99 \%$ & Un-identified & \\
\hline 185 & C. albidus & $100 \%$ & C. laurentii & $99 \%$ \\
\hline
\end{tabular}


Abu-Mejdad et al. / Basrah J. Agric. Sci., 33(1): 39-49, 2020

\begin{tabular}{|c|c|c|c|c|}
\hline 169 & Cutaneotrichosporon dermatis & $100 \%$ & Trichosporon asahii & $88 \%$ \\
\hline 36 & Cystobasidium benthicum & $99 \%$ & Un-identified & \\
\hline 71 & C. benthicum & $100 \%$ & Un-identified & \\
\hline 152 & C. benthicum & $99 \%$ & Un-identified & \\
\hline 18 & C. minutum & $98 \%$ & Un-identified & \\
\hline 81 & Debaryomyces hansenii & $100 \%$ & Candida famata & $99 \%$ \\
\hline 132 & D. hansenii & $100 \%$ & C. famata & $99 \%$ \\
\hline 193 & D. hansenii & $100 \%$ & C. famata & $99 \%$ \\
\hline 201 & D. hansenii & $100 \%$ & C. famata & $99 \%$ \\
\hline 208 & D. hansenii & $100 \%$ & C. famata & $99 \%$ \\
\hline 235 & D. hansenii & $100 \%$ & C. famata & $99 \%$ \\
\hline 255 & D. hansenii & $100 \%$ & C. famata & $99 \%$ \\
\hline 256 & D. hansenii & $100 \%$ & C. famata & $99 \%$ \\
\hline 262 & D. hansenii & $100 \%$ & Candida lipolytica & $92 \%$ \\
\hline 80 & Filobasidium oeirense & $100 \%$ & C. laurentii & $91 \%$ \\
\hline 28 & Galactomyces pseudocandidum & $100 \%$ & C. famata & $95 \%$ \\
\hline 63 & Galactomyces reessii & $100 \%$ & C. famata & $95 \%$ \\
\hline 70 & Geotrichum candidum & $100 \%$ & C. famata & $95 \%$ \\
\hline 163 & G. candidum & $100 \%$ & C. famata & $95 \%$ \\
\hline 78 & Hanseniaspora uvarum & $100 \%$ & Un-identified & \\
\hline 213 & H. uvarum & $100 \%$ & Un-identified & \\
\hline 2 & Lodderomyces elongisporus & $100 \%$ & C. famata & $95 \%$ \\
\hline 136 & L. elongisporus & $100 \%$ & C. famata & $95 \%$ \\
\hline 46 & Meyerozyma caribbica & $100 \%$ & Un-identified & \\
\hline 47 & M. caribbica & $100 \%$ & Un-identified & \\
\hline 55 & M. caribbica & $100 \%$ & Un-identified & \\
\hline 124 & Naganishia adeliensis & $99 \%$ & C. famata & $95 \%$ \\
\hline 108 & N. albida & $100 \%$ & C. albidus & $96 \%$ \\
\hline 180 & N. albida & $100 \%$ & C. laurentii & $95 \%$ \\
\hline 241 & N. albida & $100 \%$ & C. albidus & $96 \%$ \\
\hline 129 & N. albidosimilis & $99 \%$ & C. laurentii & $97 \%$ \\
\hline 65 & N. diffluens & $100 \%$ & C. albidus & $93 \%$ \\
\hline 116 & N. diffluens & $100 \%$ & C. laurentii & $95 \%$ \\
\hline 138 & N. diffluens & $100 \%$ & C. laurentii & \\
\hline 198 & N. diffluens & $100 \%$ & C. albidus & $93 \%$ \\
\hline 261 & N. diffluens & $100 \%$ & C. albidus & $93 \%$ \\
\hline 204 & N. diffluens & $100 \%$ & C. albidus & $93 \%$ \\
\hline 69 & N. liquefaciens & $100 \%$ & C. laurentii & $91 \%$ \\
\hline 137 & N. liquefaciens & $100 \%$ & Un-identified & \\
\hline 189 & N. vishniacil & $100 \%$ & C. albidus & $91 \%$ \\
\hline 154 & Pichia fermentans & $99 \%$ & Candida krusei & $93 \%$ \\
\hline 195 & P. fermentans & $100 \%$ & C. krusei & $94 \%$ \\
\hline 200 & P.fermentans & $99 \%$ & C. krusei & $93 \%$ \\
\hline 3 & Rhodotorula diobovata & $100 \%$ & C. laurentii & $94 \%$ \\
\hline 53 & R. diobovata & $100 \%$ & C. laurentii & $94 \%$ \\
\hline 141 & R. diobovata & $100 \%$ & C. laurentii & $94 \%$ \\
\hline 33 & R. mucilaginosa & $100 \%$ & C. albidus & $93 \%$ \\
\hline 82 & R. mucilaginosa & $100 \%$ & C. albidus & $93 \%$ \\
\hline 111 & R. mucilaginosa & $100 \%$ & C. albidus & $93 \%$ \\
\hline 139 & R. mucilaginosa & $99 \%$ & C. albidus & $93 \%$ \\
\hline
\end{tabular}


Abu-Mejdad et al. / Basrah J. Agric. Sci., 33(1): 39-49, 2020

\begin{tabular}{|c|c|c|c|c|}
\hline 144 & R. mucilaginosa & $100 \%$ & C. albidus & $93 \%$ \\
\hline 148 & R. mucilaginosa & $100 \%$ & C. albidus & $93 \%$ \\
\hline 159 & R. mucilaginosa & $100 \%$ & C. albidus & $93 \%$ \\
\hline 187 & R. mucilaginosa & $100 \%$ & C. albidus & $93 \%$ \\
\hline 194 & R. mucilaginosa & $100 \%$ & C. albidus & $93 \%$ \\
\hline 196 & R. mucilaginosa & $100 \%$ & C. albidus & $93 \%$ \\
\hline 210 & R. mucilaginosa & $100 \%$ & C. albidus & $93 \%$ \\
\hline 216 & R. mucilaginosa & $100 \%$ & C. albidus & $93 \%$ \\
\hline 237 & R. mucilaginosa & $100 \%$ & C. albidus & $93 \%$ \\
\hline 245 & R. mucilaginosa & $100 \%$ & Rhodotorula glutinis & $94 \%$ \\
\hline 265 & R. mucilaginosa & $100 \%$ & C. laurentii & $95 \%$ \\
\hline 219 & Symmetrospora folicola & $100 \%$ & Un-identified & \\
\hline 174 & Torulaspora delbrueckii & $100 \%$ & Un-identified & \\
\hline 177 & T. delbrueckii & $100 \%$ & Un-identified & \\
\hline 270 & T. delbrueckii & $100 \%$ & Un-identified & \\
\hline 105 & Vishniacozyma carnescens & $100 \%$ & Un-identified & \\
\hline 224 & Wickerhamomyces anomalus & $100 \%$ & Un-identified & \\
\hline 155 & W. onychis & $100 \%$ & Un-identified & \\
\hline 1 & Yarrowia lipolytica & $100 \%$ & Malassezia furfur & $97 \%$ \\
\hline 26 & Y. lipolytica & $100 \%$ & M. furfur & $97 \%$ \\
\hline 29 & Y. lipolytica & $100 \%$ & Candida & $97 \%$ \\
\hline 90 & Y. lipolytica & $100 \%$ & M. furfur & $97 \%$ \\
\hline 92 & Y. lipolytica & $100 \%$ & M. furfur & $97 \%$ \\
\hline 100 & Y. lipolytica & $100 \%$ & M. furfur & $97 \%$ \\
\hline 110 & Y. lipolytica & $100 \%$ & M. furfur & $97 \%$ \\
\hline 112 & Y. lipolytica & $100 \%$ & M. furfur & $97 \%$ \\
\hline 147 & Y. lipolytica & $100 \%$ & M. furfur & $97 \%$ \\
\hline 165 & Y. lipolytica & $100 \%$ & M. furfur & $97 \%$ \\
\hline 176 & Y. lipolytica & $100 \%$ & M. furfur & $97 \%$ \\
\hline 222 & Y. lipolytica & $100 \%$ & M. furfur & $97 \%$ \\
\hline 227 & Y. lipolytica & $100 \%$ & M. furfur & $97 \%$ \\
\hline 230 & Y. lipolytica & $100 \%$ & M. furfur & $97 \%$ \\
\hline 238 & Y. lipolytica & $100 \%$ & M. furfur & $97 \%$ \\
\hline 243 & Y. lipolytica & $100 \%$ & M. furfur & $97 \%$ \\
\hline 244 & Y. lipolytica & $100 \%$ & M. furfur & $97 \% \%$ \\
\hline 248 & Y. lipolytica & $100 \%$ & M. furfur & $97 \%$ \\
\hline 259 & Y. lipolytica & $100 \%$ & M. furfur & $97 \%$ \\
\hline 263 & Y. lipolytica & $100 \%$ & M. furfur & $97 \%$ \\
\hline 264 & Y. lipolytica & $100 \%$ & M. furfur & $97 \%$ \\
\hline 266 & $Y$. lipolytica & $100 \%$ & M. furfur & $97 \%$ \\
\hline
\end{tabular}

\section{Discussion}

Diazonium Blue B (DBB) test to detect basidiomycetes yeasts on solid media

The DBB test has been used by yeast taxonomists to distinguish between ascomycetous and basidiomycetous fungi.
During the current study, the most cells of basidiomycetous yeasts showed pink to red or violet colour reactions (+) against DBB, even when using many solid growth media, while 
ascomycetes yeast presented brown colour (-) results.

Robert et al. (2015) stated that the DBB colour reaction (reagent) is used as a primary assistant factor in differentiation between basidiomycetous (especially those with unknown teleomorph) and ascomycetous yeasts.

This test is influenced by four factors (temperature of reagent, type of culture medium that used with the reagent, incubation period of tested yeast and $\mathrm{pH}$ of culture media, which are used during the test). However, an experienced technician will implements this test more advantageous than other workers who performs a routine identification (Kregervan Rij \& Veenhuis, 1971). A neutral $\mathrm{pH}$ $(\approx 7.0)$ of medium was more crucial for a positive DBB colour reaction (Singh \& Kaur, 2018). There was considerable variation between isolates in their colour intensity (graduated from orange, pink and red) and the age of culture, which was necessary for obtaining a positive reaction (Rodrigues \& Fonseca, 2003). In our findings, most environmental yeasts were required incubation period from 7-14 days to give positive result occurrence, while a few genera like $R$. mucilignosa and $W$. anomalus showed a positive results after $24 \mathrm{~h}$.

\section{VITEK 2 compact system}

The VITEK biochemical procedures of yeasts (YBC) led to quick identification, especially for clinical specimens. Moreover, these methods are still helpful for primary identification of ecological specimens. During this study, a total of 112 yeast isolates were tested by VITEK 2 compact system but only $8.2 \%$ of them were identified accurately, whereas, $91.8 \%$ of them were not. According to this high percentages of improper identification of many isolates, it can be clearly stated that, this test is unreliable for ecological yeasts compared to the clinical isolates, especially for common yeasts. This finding is correspondent with the results of Westblade $e t$ al. (2013). Anyhow, the VITEK yeast biochemical system may be used to identify the common yeasts i.e., Candida tropicalis, $C$. parapsilosis, Candida glabrata, C. albicans and Cryptococcus neoformans with a period of twenty-four hours $(93 \%$ of precise identifications). The ability of the YBC system to determine these common isolates quickly due to an automated system, which is used in several clinical laboratories (Zhao et al., 2017). Our results confirmed that the repeated improper identification for many isolates i.e. Yarrowia lipolytica, Debaryomyces hansenii, Candida tropicalis, Rhodotorula mucilignosa etc. were in accordance with the findings of Dooley et al. (1994). Delayed biochemical reactions in VITEK system suggested that unreliable confirmation for the YBC and validation of identification by molecular technique (Valenza et al., 2008). In addition, the YBC may result in improper identification due to delayed growth of yeasts (more thanseven days), especially where workers in microbiological laboratories have less professional mycological expertise and did not give enough time to yeasts to grow before starting the biochemical test. Similar explanation was reported by Freydiere et al. (2001). Furthermore, differences among these studies, may be attributed to the laboratory conditions (in equipment, reagents, or personnel skill, old version of ID card of VITEK 2 compact system) that also can lead to misidentification .

\section{Genetic identification}

Yeast identification by traditional (morphological and biochemical) methods is a complex, difficult and time-consuming 
process that requires the high expertise and skills of the people concerned in this area. Sometimes, they give wrong or confused and incomplete identification results, such as identification to levels less than species. Khattab et al. (2016) reported that there is a need to use 90 chemical tests to bring the yeast species closer to the precise identification. In recent decades, researchers have been using molecular identification methods, which are characterized by their accuracy and rapidity in these methods, yeast strains can be isolated from substrate and identified to the species level (Alsohaili \& Bani-Hasan, 2018).

Franco-Duarte et al. (2019) reported that polyphasic approach involves multiple identification methods, including phenotypic, biochemical and genetic approaches, which are altogether can provide a comprehensive and accurate identification.

\section{Conclusion}

The Diazonium blue B was an excellent stain for distinguishing between ascomycetous and basidiomycetous yeast. While, the VITEK 2 compact system is not recommended for the identification of ecological yeast strains in comparison to the molecular technique that was perfect.

\section{Acknowledgements}

This work was performed by the help of cell research centre, Department of Biology, College of Science, University of Basrah for providing the equipment we are gratefully acknowledged.

\section{Conflict of interest}

The authors declare that they have no conflict of interest.

\section{Ethical approval}

All applicable institutional, national and international guidelines for the care and use of animals were followed.

\section{References}

Alsohaili, S.A. \& Bani-Hasan, B.M. (2018). Morphological and molecular identification of fungi isolated from different environmental sources in the Northern Ebvastern Desert of Jordan. Jordan J. Biol. Sci., 11(3): 329-337. http://jjbs.hu.edu.jo/vol11.htm

Bellemain, E.; Carlsen, T.; Brochmann, C.; Coissac, E.; Taberlet, P. \& Kauserud, H. (2010). ITS as an environmental DNA barcode for fungi: an in silico approach reveals potential PCR biases. BMC Microbiology, $\quad 10(1)$ : 189. https://doi.org/10.1186/1471-2180-10-189

Dooley, D.; Beckius, M.L. \& Jeffrey, B.S. (1994). Misidentification of clinical yeast isolates by using the updated Vitek Yeast Biochemical Card. J. Clin. Microbiol., 32(12): 2889-2892. https://www.ncbi.nlm.nih.gov/pmc/articles /PMC264196/

Dufour, J.; Verstrepen, K.; Derdelinckx, G., Boekhout, T. \& Robert, V. (2003). Yeasts in Food-Beneficial and Detrimental Aspects: Woodhead Publishing: New York: 512pp.

Franco-Duarte, R.; Černáková, L.; Kadam, S.; S. Kaushik, K.; Salehi, B.; Bevilacqua, A. \& Leszczewicz, M. (2019). Advances in chemical and biological methods to identify microorganisms-from past to present. Microorganisms, $\quad$ 7(5) $1-130$. https://doi.org/10.3390/microorganisms705 0130 
Freydiere, A.-M.; Guinet, R. \& Boiron, P. (2001). Yeast identification in the clinical microbiology laboratory: Phenotypical methods. Sabouraudia, 39(1): 9-33. https://doi.org/10.1080/mmy.39.1.9.33

Hagler, A.N. \& Ahearn, D. (1981). Rapid diazonium blue $B$ test to detect basidiomycetous yeasts. Int. J. Syst. Evol. Microbiol., $\quad 31(2)$ : 204-208. https://doi.org/10.1099/00207713-31-2204

Hierro, N.; Gonzalez, A.; Mas, A. \& Guillamón, J. (2004). New PCR-based methods for yeast identification. J. Appl. Microbiol., $\quad$ 97(4): 792-801. https://doi.org/10.1111/j.1365-

2672.2004.02369.x

Khattab, S.M.R.; Abdel-Hadi, A.M.; AboDahab, N.F. \& Atta, O.M. (2016). Isolation, characterization, and identification of yeasts Associated with foods from Assiut City, Egypt. British Microbiol. Res. J., 13(1)

:1-10. https://doi.org/10.9734/BMRJ/2016/24170

Kreger-van Rij, N. \& Veenhuis, M. (1971). A comparative study of the cell wall structure of basidiomycetous and related yeasts. Microbiology, $\quad 68(1)$ : $\quad 87-95$. https://www.microbiologyresearch.org/content /journal/micro/10.1099/00221287-68-187; jsessionid=XzcjctX3N0rMq4P2m1gdKUIU .mbslive-10-240-10-108

Latouche, G.N.; Daniel, H.; Lee, O.C.; Mitchell, T.G.; Sorrell, T.C. \& Meyer, W. (1997). Comparison of use of phenotypic and genotypic characteristics for identification of species of the anamorph genus Candida and related teleomorph yeast species. J. Clin. Microbiol., 35(12): 31713180.

https://www.ncbi.nlm.nih.gov/pmc/articles /PMC230143/
Magliani, W.; Conti, S.; Gerloni, M.; Bertolotti, D. \& Polonelli, L. (1997). Yeast killer systems. Clin. Microbiol. Rev., 10(3): 369-400.

https://www.ncbi.nlm.nih.gov/pmc/articles /PMC172926/

Robert, V.; Cardinali, G. \& Casadevall, A. (2015). Distribution and impact of yeast thermal tolerance permissive for mammalian infection. BMC Biol., 13(1): 118 https://doi.org/10.1186/s12915-0150127-3

Rodrigues, M.G., \& Fonseca, A. (2003). Molecular systematics of the dimorphic ascomycete genus Taphrina. Int. J. Syst. Evol. Microbiol., 53(2): 607-616. https://doi.org/10.1099/ijs.0.02437-0

Rodrigues, A., Pinheiro, R., Costa, J., Santos, J., Poli, J., Rosa, C. \& Nóbrega, M. (2018). Comparison between morphophysiological and molecular methods for the identification of yeasts isolated from honey. Int. Food Res. J.1, 25(1):418-422. https://docplayer.net/103197739-

Comparison-between-

morphophysiological-and-molecularmethods-for-the-identification-of-yeastsisolated-from-honey.html

Schmitt, M.J. \& Breinig, F. (2002). The viral killer system in yeast: from molecular biology to application. FEMS Microbiol. Rev., 26(3): 257-276. https://doi.org/10.1111/j.15746976.2002.tb00614.x

Singh, R. \& Kaur, N. (2018). Biochemical and molecular characterization of a new pullulan producer Rhodosporidium paludigenum PUPY-06. J. Appl. Biol. Biotechnol.,6(1):28-37. https://doi.org/10.7324/JABB.2018.60106 
Tekpinar, A.D. \& Kalmer, A. (2019). Utility of various molecular markers in fungal identification and phylogeny. Nova Hedwigia, 108(3-4): 3-4. https://doi.org/ 10.1127/nova_hedwigia/2019/0528

Török, T. \& King, A. (1991). Comparative study on the identification of food-borne yeasts. Appl. Environ. Microbiol., 57(4): 1207-1212. https://www.ncbi.nlm.nih.gov/ pmc/articles/PMC182869/

Valenza, G.; Strasen, J.; Schäfer, F., Frosch, M., Kurzai, O., \& Abele-Horn, M. (2008). Evaluation of new colourcolourimetric Vitek 2 yeast identification card by use of different source media. J. Clin. Microbiol., 46(11): $\quad$ 3784-3787. https://doi.org/10.1128/JCM.01318-08

Van der Walt, J. \& Hopsu-Havu, V. (1976). A colour reaction for the differentiation of ascomycetous and hemibasidiomycetous yeasts. Anton. Leeuw., 42(1-2): 157-163. https://doi.org/10.1007/BF00399460

Westblade, L.F.; Jennemann, R.; Branda, J.A.; Bythrow, M.,;Ferraro, M.J.; Garner, O.B. \& Mochon, A.B. (2013). Multicenter study evaluating the Vitek MS system for identification of medically important yeasts. J. Clin. Microbiol., 51(7): 22672272. https://doi.org/10.1128/JCM.0068013

Zhao, Y.; Tsang, C.; Xiao, M.; Chan, J.F.; Lau, S.; Kong, F. \& Woo, P.C. (2017). Yeast identification by sequencing, biochemical kits, MALDI-TOF MS and rep-PCR DNA fingerprinting. Med. Mycol., 56(7): 816827. https://doi.org/10.1093/mmy/myx118.

\section{تقرير جديد عن طرق تحديل سلالات الخميرة القاتلة}

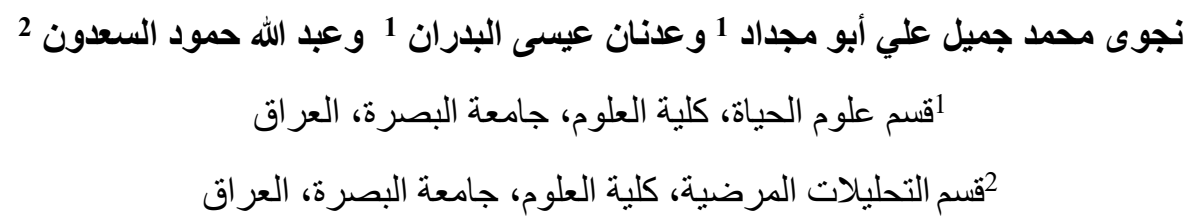

المستخلص: اجريت هذه الدر اسة لعزل وتحديد الخمائر القاتلة من عينات التربة التي جمعت من مو اقع مختلفة في محافظتي البصرة

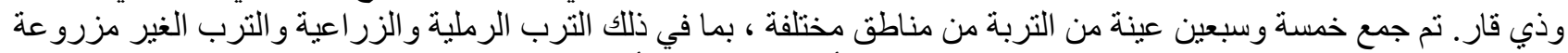

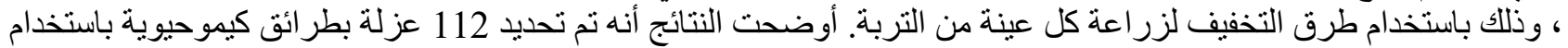

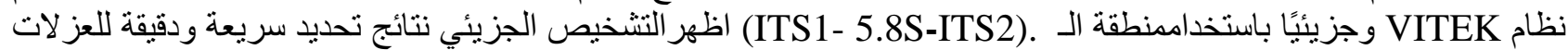

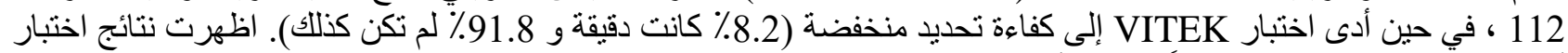

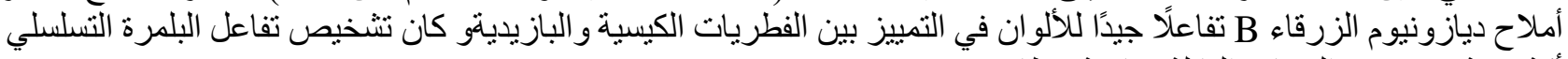

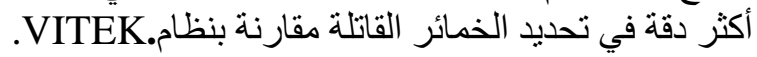
الكلمات المفتاحية: املاح الديازونيم الزرقاء B , الفايتك, تفاعل البلمرة التسلسلي,تثخيص الخمائر القاتلة . 